\title{
Semelhantemente a pessoas...
}

\author{
Harley E. A. Bicas, Cristina Muccioli, Mauro S. O. Campos, Paulo E. C. Dantas
}

Semelhantemente a pessoas e outros seres vivos, instituições e empresas também possuem um ciclo vital. Nascem e, várias delas, por falta de boa estruturação ou por suas más formações, não duram muito tempo. Outras crescem e amadurecem, têm registro de identificação, tornam-se fortes e respeitadas, passam por dificuldades, ficam doentes, tudo como se fossem pessoas. Tanto que até se dá a elas o nome de "pessoas jurídicas". Se pertencem a um universo acadêmico, como as revistas de publicações científicas, estão igualmente sujeitas a julgamentos e avaliações, recebem reconhecimentos por suas conquistas e méritos, desenvolvem um currículo. Adaptam-se aos tempos.

Os Arquivos Brasileiros de Oftalmologia, publicação científica oficial do Conselho Brasileiro de Oftalmologia e por este adotada (em 1977, volume 40, a partir do n⿳ำ 4), após 39 anos de serviços orgulhosamente prestados à ciência e à nação (desde sua fundação em 1938), não tem como se queixar de sua saúde. Ao contrário, com suas tiragens continuamente ascendentes (nossos mais recentes números são imprimidos em 7400 cópias), com sua divulgação também dinamizada pelos meios eletrônicos disponíveis (vários endereços de acesso), com suas memórias cada vez mais recuperadas (pelo menos a partir de $1986 \mathrm{em}$ discos compactos), com reconhecimento de valor pelas mais renomadas agências internacionais de acompanhamento de revistas científicas, e recebendo uma crescente demanda para publicação de matérias, demonstra grande vigor. Tudo resulta do inequívoco progresso da oftalmologia brasileira, da competência de seus autores e do importante protagonismo de Editores, Editores Associados e membros de nosso Conselho Editorial: selecionando, julgando, corrigindo, revisando, enfim, trabalhando com grande dedicação e afinco para disponibilizar, a cada dois meses, o que de melhor nos chega.

Certamente, essa equipe requer alterações periódicas. Quase sempre porque desempenhos em favor da revista, graciosamente ofertados, podem se tornar momentaneamente mais difíceis. A mudança de uma posição a outra não tem o significado de promoção ou de rebaixamento e só deve ser interpretada como assunção de responsabilidades maiores ou menos desgastantes: há e sempre haverá um profundo respeito aos trabalhos realizados por cada célula desse organismo. Às vezes nem sequer aparente mas, não obstante, profundamente importante.

E voltando ao assunto inicial, nossa revista, semelhantemente a pessoas, faz também, ao fim de cada ano, seus agradecimentos especiais a amigos e colaboradores que tanto contribuíram à sua vitalidade. Aliás, reserva-se o primeiro número de cada ano para relacionar e reverenciar pessoas que atuaram de modo muito efetivo no julgamento de mérito dos artigos recebidos para publicação e cujos nomes não constam da lista de Conselheiros Editoriais. Aliás, convém ressaltar que não cabe qualquer distinção de valor ou hierarquia entre estes e aquelas, já que a escolha de um julgador, embora sempre se faça com o pressuposto de sua competência na matéria sobre a qual é convidado a se manifestar, muito frequentemente fica condicionado à disponibilidade de tempos (para julgamentos) e a oportunidades. É mesmo fato que alguns dos nomes abaixo relacionados, Conselheiros Editoriais "ad hoc", foram mais acionados e trabalharam mais do que alguns dos nomes oficialmente dados como de Conselheiros Editoriais "efetivos".

Assim, com nossos sinceros e efusivos agradecimentos, em nome da oftalmologia brasileira, damos a público a relação desses, antes, anônimos Conselheiros Editoriais que, a convite, prestaram importantíssimas contribuições no julgamento de matérias que nos foram enviadas para publicação:
A. Duarte
Abelardo Couto Jr.
Acacio Alves de Souza Lima Filho
Adriana Santos Forseto
Áisa Haidar Lani
Amélia Kamegasawa
Ana Estela Besteti Pires Ponce Sant'Anna
André Messias
André Romano
Arnaldo Furman Bordon
Belquiz Rodrigues A. Nassaralla
Breno Barth
Bruno Castelo Branco
Carlos Eduardo Leite Arieta
Carlos Roberto Neufeld
Carlos Teixeira Brandt
Celina Tamaki Monteiro de Castro
Christiane Rolim de Moura
Cintia S. Paiva
Claudia Maria Francesconi
Claudio Alberto Magalhães da Silveira
Clelia Maria Erwenne
Cleusa Coral-Ghanem
Consuelo Bueno Diniz Adan
Davi Araf
Denise Fornazari de Oliveira
Denise Vuono Chinzon
Diane Ruschel Marinho
Edmea Rita Temporini
Edmundo Frota de Almeida Sobrinho
Edson Procianoy 
Edson Shizuo Mori

Eduardo Ferrari Marback

Eliana Aparecida Forno

Enyr Saran Arcieri

Fausto Uno

Fernando Arevalo

Francisco Assis Cordeiro Barbosa

Francisco Eduardo Lopes de Lima

Galton Carvalho Vasconcelos

Ivan Maynart Tavares

Jair Giampani Jr

Jayter Silva de Paula

João Amaro Ferrari Silva

João Borges Fortes Filho

João Carlos Miranda Gonçalves

João J. Nassaralla Jr.

Joaquim Marinho de Queiróz

Jonathan Clive Lake

José Carlos Eudes Carani

José Vital Filho

Joyce Hisae Yamamoto

Juliana Maria Ferraz Sallum

Kimble Matos

Laurentino Biccas Neto

Lincoln Leme Freitas

Luciana Peixoto Finamor

Luís Antonio Vieira

Luís Paves

Luiz Alexandre Rassi Gabriel

Magno Antonio Ferreira

Maira Saad de Ávila Morales

Marcelo Francisco Gaal Vadas

Marcelo Hatanaka

Marcelo Jordão Lopes da Silva

Marcia Keiko Uyeno Tabuse

Marcos Carvalho da Cunha

Maria Aparecida Onuki Haddad

Maria Auxiliadora M. Frazão Sibinelli

Maria Emilia Xavier dos Santos Araújo

Maria Teresa Bonanomi

Mário Teruo Sato

Marivaldo Castro de Oliveira
Mariza Aparecida Polati

Mariza Toledo de Abreu

Marlon Moraes Ibrahim

Marta Beatriz Filippi Sartori

Martha Maria Motono Chojniak

Maurício Bastos Pereira

Maurício Maia

Mauro Nishi

Mauro Waiswol

Midori Osaki

Mônica de C. Alves Paula

Mônica Fialho Cronemberger

Moysés Zajdenweber

Myrna Serapiao dos Santos

Newton Kara-José Jr.

Niro Kasahara

Norma Helen Medina

Nubia Cristina Freitas Maia

Patrícia Mitiko Santello Akaishi

Paulo Afonso Batista dos Santos

Paulo Gilberto Jorge Fadel

Renata Attanasio de Rezende

Renato Ambrósio Jr.

Ricardo Suzuki

Roberto Pedrosa Galvão Filho

Roberto Pinto Coelho

Rodrigo Pessoa Cavalcanti Lira

Ronaldo Boaventura Barcellos

Rosa Maria Graziano

Rosana Nogueira Pires da Cunha

Rui Barroso Schimiti

Ruth Miyuki Santo

Sergio Burnier

Simone Finzi

Solange Rios Salomão

Tomás Fernando Scalamandré Mendonça

Vera Lúcia D. Monte Mascaro

Victor Evangelista de Faria Ferraz

Virgilio Centurion

Wagner Zacharias

Wener Cella Passarinho

Wilson de Freitas 